\title{
La mémoire organisationnelle en rupture avec la mémoire individuelle
}

André A. Lafrance

\section{(2) OpenEdition}

1 Journals

Édition électronique

URL : http://journals.openedition.org/communicationorganisation/2895

DOI : 10.4000/communicationorganisation.2895

ISSN : $1775-3546$

Éditeur

Presses universitaires de Bordeaux

Édition imprimée

Date de publication : 1 mai 2004

ISSN : 1168-5549

Référence électronique

André A. Lafrance, "La mémoire organisationnelle en rupture avec la mémoire individuelle »,

Communication et organisation [En ligne], 24 | 2004, mis en ligne le 19 décembre 2012, consulté le 19 avril 2019. URL : http://journals.openedition.org/communicationorganisation/2895 ; DOI : 10.4000/ communicationorganisation.2895

Ce document a été généré automatiquement le 19 avril 2019.

(c) Presses universitaires de Bordeaux 


\title{
La mémoire organisationnelle en rupture avec la mémoire individuelle
}

\author{
André A. Lafrance
}

1 Le développement des technologies de l'information a pu laisser croire qu'il était possible d'informatiser la mémoire organisationnelle. Retrouvant ainsi, étrangement, les illusions de Weber on espérait pouvoir dépersonnaliser cette mémoire en lui attribuant des mécanismes centralisés d'enregistrement et en supposant une réactivation quasiautomatique des éléments pertinents de cette mémoire à chaque fois que les événements sollicitent une réorientation, mineure ou majeure, des opérations d'une organisation.

2 Cette conception de la mémoire collective, comme garante de l'identité organisationnelle répond clairement à une certaine vision des intendances informatiques des entreprises. Mais est-elle cohérente avec les recherches sur le fonctionnement de la mémoire individuelle dont elle prétend être l'héritière et dont elle cherche à transposer les éléments dans la construction d'une "personnalité » organisationnelle? Les recherches actuelles sur les logiciels supportant le travail coopératif témoignent d'une nouvelle sensibilité des milieux informatiques à la modulation des apports individuels.

3 Il ne s'agit pas néanmoins, d'un phénomène nouveau. En 1976. G.R. Loftus et E.F. Loftus avaient déjà proposé que la faculté de mémorisation ne-soit pas limitée aux humains, mais qu'elle existe pour plusieurs objets. Nous sommes, ici dans le domaine des analogies qui font de l'organisation une entité biologique jouissant des propriétés d'un être humain. Et l'une de ces propriétés serait justement cet instrument de la gouvernance individuelle qu'est la mémoire. Même si l'application du concept de mémoire au niveau de l'organisation ne fait pas consensus au sein des chercheurs, nous allons maintenir, pour les fins de notre exposé, le cap sur cette analogie. Elle a déjà bien servi l'élaboration d'une infrastructure conceptuelle pour les discours des théoriciens de la communication organisationnelle.

4 Le présent exposé se situe dans un projet de recherche visant à fournir aux acteurs et aux intervenants des instruments pour observer les éléments mémorisés dans et par l'organisation. ${ }^{1}$ Nous nous proposons d'étudier comment les conditions actuelles de 
fonctionnement des entreprises commerciales attirent à nouveau l'attention sur la mémoire organisationnelle et influencent ses modes de création et d'utilisation. Et nous espérons apporter notre contribution à la construction d'une grille d'analyse permettant aux chercheurs de porter un diagnostique sur le fonctionnement d'une mémoire organisationnelle et d'aider les acteurs à se prescrire, s'il y a lieu, un remède à toute dysfonction.

\section{La mémoire-action}

Pour suivre le cheminement stratégique de cette mémoire collective dont serait dotée l'organisation, il faut remonter à la source de l'analogie, la mémoire individuelle. Et pour ce faire, nous relire quelques études-phares portant sur le sujet et tenter d'en appliquer les résultats à la mémoire organisationnelle. À quoi sert cette mémoire dans la gouvernance de l'individu? Une croyance largement répandue est que l'acquisition, la rétention et la récupération de connaissances et d'expériences depuis les dépôts de la mémoire, influencent le comportement futur de l'individu.

6 Ainsi la mémoire servirait à interpréter un stimulus, à offrir des comportementsréactions possibles, à évaluer la pertinence de ces recomportements par rapport à l'interprétation du stimulus. Elle servirait aussi à imaginer, si nécessaire, un nouveau comportement en groupant, de façon différente, des éléments d'anciens comportementsréactions. Et finalement, elle permettrait de conjuguer les habiletés nécessaires pour réaliser le recomportement ou le nouveau comportement. (Walsh et Ungson. 1991)

Dans son sens le plus simple, celle-ci fait référence à l'information à propos du passé d'une organisation qui peut, comme nous venons de le voir pour l'individu, être récupéré dans le présent pour influencer une prise de décision ou un comportement. Mais ce processus de récupération n'est qu'une opération en aval de ce qui doit se passer en amont pour alimenter cette mémoire. Nous y reviendrons.

Plusieurs n'hésitent pas à faire de cette mémoire l'un des moteurs de la réactivité de l'entreprise. C'est en puisant, de façon stratégique, dans le contenu de cette mémoire qu'elle peut planifier ses réponses aux défis quotidiens présentés pur un environnement en mutations accélérées et successives, fa métaphore du traitement de l'information au niveau individuel est ici très représentative. La récupération de l'information se traduit sur un continuum variant de la récupération automatique à la récupération contrôlée, La récupération automatique consiste à la connaissance intuitive et sans effort d'une information pertinente à un stimulus décisionnel actuel faisant partie d'une séquence d'actions bien établie dans l'organisation, Une récupération contrôlée fait, quant à elle, référence à une recherche consciente d'une information en faisant référence à une décision passée. Cette démarche nécessite un effort supplémentaire et ne peut être effectuée que pur un individu ou une collectivité d'individus.

Dans sa forme la plus dépouillée de toute innovation, la récupération prend la forme d'une routine. Les routines sont différenciées d'une procédure d'opération standard par le l'ait qu'elles ne sont pas explicitement et officiellement répertoriées dans l'organisation. Par ailleurs, elles sont difficiles à saisir car les acteurs ne prennent généralement pas conscience des gestes posés et de l'utilité de ces derniers. Elles émergent souvent à travers un apprentissage graduel entre les acteurs dans un contexte 
historique qui peut parfois rendre difficile la compréhension et la réforme de celle-ci. (Cohen et Bacdayan. 1994)

10 Si la composition d'une action nécessite une certaine dose d'innovation par rapport à une routine déjà établie, elle se base sur la dérivation d'un comportement bien rodé. Trois niveaux d'improvisation existent et se distinguent par le degré de changement effectué par rapport à la référence de-base. Ils varient d'un simple ajustement de la routine à un remodelage complet d'où émergent de nouveaux schémas (Moorman et Miner. 1998). Le simple ajustement d'une improvisation, née d'un mélange de bricolage, de créativité et d'intuition, peut mener à une adaptation, définie comme l'ajustement d'un système aux conditions extérieures. Si elle est réussie, l'adaptation peut mener à un troisième niveau d'improvisation, l'apprentissage, processus qui implique la découverte, la rétention et l'utilisation ultérieure des connaissances ainsi mémorisées.

\section{La mémoire-contention}

11 On a aussi attribué à cette mémoire de nombreuses responsabilités - pour ne pas dire "culpabilités» - dans le manque de réactivité de certaines grandes entreprises. La mémoire était alors considérée comme un carcan culturel retardant, ou même interdisant, une interprétation proactive des signaux envoyés par l'environnement. Cela convient bien au terme contention décrite comme « un appareil ou procédé destiné à immobiliser dans un but thérapeutique». Car c'est au nom d'une certaine mémoire organisationnelle témoin d'heures glorieuses, et des comportements dont on prétend qu'elle garantit le succès, qu'on s'oppose aux différentes improvisations proposées par des acteurs de l'organisation.

12 C'est à cette mémoire-contention que doivent habituellement s'attaquer les gestionnaires prenant la direction de grandes entreprises en train de perdre pied dans un marché aux nouveaux contours concurrentiels. Deux héros du monde nord-américain des affaires. Louis V. Gerstner Jr (IBM) et Jack Welch (GE) ont. par leurs discours et leurs écrits, donné ses lettres de-noblesse à la dénonciation de cette mémoire-contention.

13 Gerstner (2002) a donné à ses mémoires le titre de: "Qui a dit que les éléphants ne pouvaient pas danser? » Tout un programme ! Noël M. Tichy et Stratford Sherman (1994 : 37) ont étudié la révolution apportée par Weleh dans la gestion de General Electric.: «Weleh croyait que les succès passés de GE contenaient la semence de difficultés présentes et futures. Il était prêt à agir selon cette perception. Dans un environnement de changement incessant, peu d'idées d'affaires demeurent utiles pour longtemps. Après un certain temps, même les concepts les plus fructueux doivent être abandonnés. Mais, chez GE un siècle d'expérience en affaires s'incarnait alors dans une culture corporative rigide et une structure organisationnelle autoritaire. »

14 Il est intéressant de noter que depuis qu'ils ont eux-mêmes cédé la place à des successeurs, leur gestion est à son tour considérée comme dépassée et leurs choix, périmés.

15 Cette mémoire-contention prêle d'ailleurs flanc à toutes sortes de délinquances. Dans son roman Le Burelain. Richard Jorif (1991 : 22) décrit bien cette ambiguïté :

«Vous vous êtes bien imprégné du règlement intérieur ?il ne faut pas du tout prendre au pied de la lettre, ça date, ça date beaucoup, et d'ailleurs tout le monde s'en balance y compris les syndicats... Je vais vous dire : ce document remonte lui 
aussi au premier temps de la société, il y a eu quelques amendements au début des années soixante, et depuis on n'y a plus touché : le président ne veut pas qu'on ravale ce monument historique. «Laissez les choses en étal » c'est sa phrase. »

\section{La mémoire-protection} de salut des entreprises emportées dans la tourmente des gestes posés par leurs gestionnaires. Des naufrageurs ont voulu solder celle identité pour financer la construction d'empires financiers hétéroclites au service de leur boulimie boursière. Ils ont créé de nouveaux Cronos avalant leurs enfants pour assurer leur développement et leur survie. Les personnels ne savaient plus pour qui - et souvent, pourquoi - ils travaillaient, lin fait, ils avaient l'impression de travailler dans un milieu victime d'un Alzheimer collectif. L'attention des gestionnaires étant dirigée vers le marché boursier et non celui des biens ou services produits, ces derniers n'avaient ni le temps ni même l'intention de contribuer à l'acquisition de ce qu'on pourrait appeler « une nouvelle mémoire vive » de l'organisation qu'ils étaient en train de mettre au monde.

Mais voilà que cette identité est en train de remonter à la surface des eaux troublées par de spectaculaires crises de trésorerie. Les délestages de secteurs complets d'activités leur redonnent une nouvelle vie qui reprend contact avec celle qu'on avait tenté de dissoudre dans des regroupements contre nature. L'identité organisationnelle tente de retrouver le contact avec les opérations d'une mémoire atrophiée par ces années de turbulence. La mémoire devient comme un incubateur pour les re-nouveaux-nés leur offrant la chaleur réconfortante

Une autre cause du regain de faveur et de ferveur pour l'identité, c'est le malaise profond causé par les comportements criminels de certains gestionnaires. Les personnels des entreprises qui ont floué leurs actionnaires ou leurs clients essaient désespérément de regagner la confiance de ces derniers en démontrant que ces comportements ne s'expliquent que la présence, tout à fait étrangère à l'identité organisationnelle d'un «virus de la cupidité » (selon l'expression d'Alan Greenspan. président de la Federal Reserve Board). Il leur apparaît donc urgent de redéfinir cette identité et surtout de la revaloriser.

\section{Trois types de mémoire}

19 Il y aurait donc plusieurs types de mémoire, en ce qui concerne leur contenu : épisodique procédurale et sémantique. Les mémoires épisodique et sémantique sont déclaratives, explicites et accessibles consciemment dans les processus de récupération contrôlée. Elles mémorisent les faits, les épisodes, les événements de la vie de tous les jours. La mémoire épisodique l'ait référence à des événements spécifiques dans l'espace-temps (« dimanche dernier, j'ai passé la soirée avec ma mère ») et la mémoire sémantique qui représente les connaissances générales emmagasinées au cours d'expériences de vie spécifiques. La mémoire procédurale est implicite et est accessible seulement à travers les performances, en engageant les habilités et les opérations dans lequel les connaissances sont incluses. (Squire. 1986)

Nous allons reprendre ces trois types et les adapter à nos besoins. La mémoire épisodique serait celle qui module le recours aux deux autres mémoires, qu'il s'agisse d'un nouveau

Communication et organisation, 24 | 2012 
comportement ou d'un recomportement en récupération automatique ou contrôlée. Elle ferait référence aux épisodes vécus pur chacun des individus à l'intérieur de l'organisation. Réservée à chacun de ces individus, lu mémoire épisodique ne saurait être collective, fille est néanmoins, organisationnelle puisqu'elle se développe dans un même cadre - l'organisation pour tous les individus œuvrant dans cette organisation et qu'elle peut être colorée, facilitée ou handicapée par cette même organisation. Le chercheur peut aussi tirer des tendances qualitatives ou des compilations quantitatives des contenus de toutes ces mémoires épisodiques.

21 La mémoire procédurale concerne la façon de faire les choses dans l'organisation. Son contenu donne lieu à des nouveaux comportements ou à des recomportements. Ce que nous avons appelé plus haut, l'improvisation, avec ses trois niveaux d'ajustement, d'adaptation et d'apprentissage, suggère de développer une méta-routine capable d'accéder aux routines en mémoire procédurale pour en faire des recombinaisons structurées.

La mémoire sémantique s'intéresse aux connaissances qui peuvent être représentées de façon symbolique et que possède une entreprise à propos du monde qui l'entoure, comme ses marchés, ses concurrents, ses clients... Ce serait ce type qui jouerait particulièrement le rôle de ce que nous avons appelé mémoire-protection...

On peut présenter les trois types comme encapsulés dans une pyramide hiérarchique. (Tulving. 1985). La mémoire épisodique au sommet, émerge, comme une catégorie spécialisée, de la mémoire sémantique puisque la façon de recourir aux deux autres mémoires dépend de la perception et de l'interprétation du monde dans lequel se produit ce recours. De même, la mémoire sémantique, comme une catégorie spécialisée de la mémoire procédurale à la base de la pyramide, puisque la perception et l'interprétation du monde pourraient être considérées comme des façons de faire de l'organisation. Il en découle que pour posséder la mémoire épisodique l'on doive obligatoirement avoir une mémoire sémantique et une mémoire procédurale. Il faut savoir comment faire (épisodique) pour répondre à une question ou un défi posés par un environnement que l'on sait interpréter (sémantique) en utilisant une façon de faire ou un mélange de façons de faire propres à l'organisation (procédurale). L'audit d'une mémoire organisationnelle doit donc tenir compte de l'état de ces trois mémoires. Si les supports de la mémoire procédurale sont relativement faciles à observer, les deux autres exigent des méthodologies assez complexes et coûteuses d'enquête, d'observation et de mise en situation. Et leur analyse implique une mise en garde contre l'interpénétration des données appartenant à chacune des mémoires, du moins en fonction des frontières opérationnelles que nous en proposons.

\section{Acquisition et rétention}

Les processus associés à la mémoire organisationnelle doivent inclure non seulement la récupération de l'information, mais aussi son acquisition et sa rétention. Nous allons voir que contrairement à la récupération et à l'utilisation des informations mémorisées, leur acquisition et leur rétention se font actuellement en complète rupture avec le fonctionnement de la mémoire individuelle.

Selon une nouvelle approche, la région corticale logeant les processus de perception, d'analyse, et de traitement de l'information à apprendre abrite également la mémoire. En ce sens, l'information en mémoire est localisée dans de petites régions définissables, 
contrairement à la vision qui prétendait que la mémoire était également distribuée à travers le cortex. En somme, chaque type d'information (visuelle, auditive, spatiale...) est crypté séparément dans le cortex. On peut toutefois dire que l'information est distribuée dans le sens que plusieurs zones du cerveau sont nécessaires pour saisir un événement dans sa globalité. (Squire. 1986).

Il est important de noter qu'à titre de "case mémoire " seul l'individu est à même de pleinement comprendre le pourquoi d'un pair stimulus décisionnel - réponse de l'organisation dans le contexte de l'histoire de l'organisation. Même pendant des tâches simples, les gens utilisent une multitude de petites mémoires redondantes qui sont distribuées dans l'environnement. Ils utilisent plusieurs artefacts qui synthétisent l'information dont ils ont besoin de manières différentes. Le téléphone, l'agenda, les logiciels et le bloc-notes sont par exemple utilisés par une secrétaire. (Ackermann et Halberstadt. 2000)

Dans une organisation, même pour la réalisation de tâches simples, on utilise une multitude de petites mémoires redondantes qui sont distribuées dans l'ensemble de la structure opérationnelle. Et on a recours à plusieurs artefacts qui synthétisent l'information, mais de manières différentes (Ackermann et Halberstadt. 2000).

\section{La rupture}

Cela pose donc la question de la contribution de chacun des membres de l'organisation à la composition et à l'utilisation de cette mémoire organisationnelle le départ à la retraite, souhaité par l'individu ou imposé par l'organisation, tient-il compte de cette contribution? Comment les organisations peuvent-elles maintenir l'identité et l'originalité qui justifient leur présence et leur survie sur le marché, alors qu'elles éliminent, pour des raisons de lactiques boursières et de fluctuations des marchés, des zones complètes du cortex organisationnel ? La réponse à ces questions semble avoir été trouvée dans l'accumulation et l'externalisation des données dans des mémoires informatiques de plus en plus puissantes. Les gestionnaires ont à leur disposition des informations tirées automatiquement de chacun des mouvements de l'organisation. Des groupes d'informations sont, souvent, transmis à des services extérieurs qui les traitent et les retournent sous des formes définis par les mandats reçus.

29 Mais si on a pu réaliser une captation des activités de l'organisation, on se retrouve avec une masse de données qu'il faut interpréter. Inversant le dicton. on se retrouve avec " une forêt qui cache l'arbre » dont le bois pourrait contribuer à la construction d'une prise de décision, fa mémoire procédurale peut probablement se satisfaire de cette situation. Ce n'est évidemment pas le cas de la mémoire épisodique essentiellement liée à l'individu. Mais ce qui est moins évident, c'est le sort de la mémoire sémantique organisationnelle dont certains éléments semblent pouvoir être récupérés et externalités. En fait, en rupture avec la mémoire individuelle, les gestionnaires gardiens de la mémoire organisationnelle semblent croire, actuellement, qu'on pourrait créer une méta mémoire formant comme un double unifié de la diversité des locus de conservation.

30 «Les physiciens ont conclu que même la masse de matière qui nous apparaît lu plus solide et la plus lourde n'est presque exclusivement que de l'espace vide. Mais à un niveau sous microscopique, des particules de matière réparties dans un grand vide ont un poids et une densité si incroyable et sont liées les uns aux autres par des forces si puissantes, qu'elles arrivent à produire ensemble toutes les propriétés du béton, du fer et de la 
pierre. De la même façon, des particules de connaissance sont réparties dans un grand vide d'ignorance. Tout dépend de la solidité de ces particules individuelles, ainsi que de la force de la liaison et de la coordination existant entre elles. » (Soweto. $1996: 4$ )

Le mouvement visant la création d'une méta mémoire a recours à divers instruments et processus d'unification des éléments mémorisés avec l'espoir de renforcer la force de la liaison et de la coordination existant entre eux. Il s'agirait non seulement de combattre l'entropie inhérente à tout système, mais aussi de délocaliser la rétention mnémonique. Car on sait très bien que ces lieux sont appelés à disparaître de façon cyclique avec les restructurations d'entreprises et de façon chronique avec le vieillissement et le départ à la retraite de leurs personnels.

Le prochain défi sera de mieux comprendre le fonctionnement de la mémoire organisationnelle et de faire un choix stratégique : le «beau risque » de la mise en réseau des mémoires individuelles ou le "coup de force " permanent de la ponction centripète des mémoires individuelles vers une mémoire organisationnelle toujours menacée d'obsolescence ou accusée d'incohérence.

\section{BIBLIOGRAPHIE}

GERSTNER. L.V.. Who Says Elephants Can't Dance? New York : Harper Business. 2002. 372 p.

JORIF. R.. Le Burelain. Paris : Editions François Bourin. coll. l'olio. 1991. 223 p.

LOTTUS. G.R.. LOFTUS. E.F.. Human memory : The processing of information. Hillsdale : N.I : Erlbaum. 1976.

SOWELL. T., Knowledge and Decisions. New York : Basic Books. 1996. 422 p.

TICHY. N.M., SHERMAN. S.. Control Your Destiny or Someone Else Will. New York : HarperBusiness. 1994. $459 \mathrm{p}$.

ACKERMAN. M.S.. HALVERSON. C.A. Reexamining organisational memory. (Communication of the ACM. 2000. 43(1). 59-64.

COHEN. MD. BACDAYAN, P., Organizational routines are stored as procedural memory: Evidence from a laboratory study. Organization Science. 1994. 5(4), 554-568.

MOORMAN. C., MINER. A.S. Organizational improvisation and organizational memory. Academy of Management Review. 1998. 23(4). 698-723.

SQUIRE. L.R., Mechanisms of memory. Science. 1986. 232(27). 1612-1619.

TULVING. E., Memory and consciousness. Psychologie Canadienne. 1985. 26(1). 1-12.

WALSH. J.P., UNGSON. G.R. Organizational memory. Academy of Management Review, 1991. 16(1). $57-91$. 


\section{NOTES}

1. Cet exposé a été construit à partir d'une recherche documentaire commandée par l'auteur et réalisée par Sacha Gagnon

\section{AUTEUR}

\section{ANDRÉ A. LAFRANCE}

Comité scientifique de la Chaire en relations publiques. (Université du Québec à Montréal). Université de Montréal 